Edición Extra-Ordinaria.

Memorias del 1 Congreso Nacional de Investigación en Enseñanza de la Biología.

VI Encuentro Nacional de Investigación en Enseñanza de la Biología y la

Educación Ambiental. ISSN 2027 1034. P. p. 495-502.

\title{
LA REVEGETALIZACIÓN COMO HERRAMIENTA PARA LA ENSEÑANZA DE LA BOTÁNICA EN EL GRADO NOVENO DEL INSTITUTO TÉCNICO INDUSTRIAL DE TOCANCIPÁ
}

\section{THE REFORESTATION LIKE TOOL FOR THE EDUCATION OF THE BOTANY IN THE DEGREE NINTH OF THE INDUSTRIAL TECHNICAL INSTITUTE OF TOCANCIPÁ}

POR: Wilson Alexander Nieto Arévalo ${ }^{1}$

\section{RESUMEN}

Durante la práctica pedagógica realizada en el Instituto Técnico Industrial de Tocancipá; se reconoce que la revegetalización de las zonas verdes de la institución contribuye al aprendizaje de la botánica a partir del cambio de actitudes de los estudiantes de noveno frente a las plantas, además de su incorporación en el plan de estudios.

La investigación intenta realizar un aporte a la enseñanza de la botánica, por lo que indaga acerca de los conocimientos botánicos de los estudiantes; a partir de un enfoque cualitativo, método descriptivo y estudio de análisis de actividades se reconocen las principales actitudes frente a la conservación de las plantas, además del conocimiento de su entorno. Finalmente se atribuyen un cambio de actitudes frente estos organismos y al mundo vivo en general, por lo que además de aportar a la enseñanza de la botánica, se contribuye a la enseñanza de la biología en Colombia a partir de la elaboración de un recurso educativo.

PALABRAS CLAVE: Revegetalización, enseñanza de la botánica, Instituto Técnico Industrial de Tocancipá, conservación

\section{ABSTRACT}

During the pedagogical practice realised in the Instituto Técnico Industrial de Tocancipá; one is clear that the reforestation of the green zones of the institution contributes to the learning of the botany from the change of attitudes of the students of ninth course in front of the plants, besides its incorporation in the curriculum.

The investigation tries to realise a contribution to the education of the botany, reason why it investigates about the botanical knowledge of the students and from a qualitative approach, descriptive method and study of analysis of activities, the

1'Wilalex_91@hotmail.com. Estudiante de Licenciatura en Biología. Universidad Pedagógica Nacional 
Bio -grafía Escritos sobre la Biología y su Enseñanza.

Edición Extra-Ordinaria.

Memorias del 1 Congreso Nacional de Investigación en Enseñanza de la Biología.

VI Encuentro Nacional de Investigación en Enseñanza de la Biología y la

Educación Ambiental. ISSN 2027 1034. P. p. 495-502.

main attitudes against the conservation of the plants are clear, besides the knowledge of their surroundings. Finally these organisms attribute themselves to a change of attitudes in front and to the alive world generally, reason why besides contributing to the education of the botany, it is contributed to the education of Biology in Colombia from the elaboration of an educative resource.

KEYWORDS: Reforestation, botany teaching, Instituto Técnico Industrial de Tocancipá, Conservation.

\section{INTRODUCCIÓN}

La enseñanza de la botánica en las instituciones educativas adquiere importancia cuando se reconoce el contexto de las mismas y las problemáticas del mundo actual en el que la conservación de las plantas y otros organismos garantiza el mantenimiento de los sistemas; es así como se establece que una de las principales falencias es la evidente falta de conciencia acerca de la conservación de las plantas endémicas en las comunidades educativas de lugares como Bogotá y municipios aledaños (en especial el municipio de Tocancipá); es posible que sea por la actual organización del currículo en las IE pues no existe una cátedra correspondiente a la enseñanza de la botánica o no se establece en los estándares propuestos para el área de Ciencias Naturales, ya que éste se centra en la enseñanza de tópicos de biología general, además de Física y Química.

$1 \quad$ En miras de hacer un aporte a dicha problemática se elabora una revisión de algunos autores que manifiestan una preocupación similar, el Norteamericano HERSHEY (1996) propone incentivar a los estudiantes a interesarse por la botánica a partir de campañas en el colegio en las cuales el foco sea el árbol nacional o la flor nacional, ya que en muchas ocasiones se observa ese patriotismo en ellos; dado que el autor es estadounidense manifiesta que "Naciones vecinas tienen árboles nacionales. Cada estado tiene un árbol estado y una flor estado. El símbolo nacional de Canadá es el arce (Acer), con una hoja de arce predominando en el centro de la bandera canadiense. El árbol nacional de México es el ciprés Montezuma (Taxodium mucronatum)

Otra posibilidad sería que todos los colegios del país arborizaran por toda la nación donde los estudiantes identificaran, inventariaran, propagaran plantas, fertilizaran, observaran y experimentaran con árboles de su campus, intercambiando información con otros colegios vía internet y haciendo tours de arborización con otros estudiantes y ciudadanos" (HERSHEY, 1996).

Estas posibilidades son interesantes, en tanto son fácilmente aplicables en países megadiversos como Colombia y en especial en el municipio de Tocancipá que se 
Bio -grafía Escritos sobre la Biología y su Enseñanza.

Edición Extra-Ordinaria.

Memorias del 1 Congreso Nacional de Investigación en Enseñanza de la Biología.

VI Encuentro Nacional de Investigación en Enseñanza de la Biología y la

Educación Ambiental. ISSN 2027 1034. P. p. 495-502.

caracteriza por un ecosistema de bosque alto andino con un gran número de especies vegetales por lo que una forma de incentivar la enseñanza de la botánica es a partir de la revegetalización y conservación de esas especies nativas presentes en las zonas aledañas a las instituciones educativas de un sector rural.

El Instituto Técnico Industrial es una institución de carácter oficial que se caracteriza por presentar amplias zonas verdes en su infraestructura además de estar ubicado en la Vereda Verganzo del municipio ya mencionado; por lo que utilizar la revegetalización de las zonas verdes de la institución como herramienta para la enseñanza de la botánica consiste en una propuesta interesante.

Con miras al desarrollo de dicha propuesta se plantean los siguientes objetivos:

\section{OBJETIVO GENERAL}

Reconocer aspectos que favorecen la enseñanza de la botánica en estudiantes de Noveno del Instituto Técnico Industrial de Tocancipá, partiendo de la conservación y la revegetalización de las zonas verdes con las que cuenta dicha institución.

\section{OBJETIVOS ESPECÍFICOS}

- Indagar acerca de aspectos que favorecen la enseñanza de la botánica en estudiantes de noveno a partir de lo que se está entendiendo por botánica en el Instituto Técnico Industrial de Tocancipá.

- Realizar actividades de conservación y revegetalización en las zonas verdes de la institución educativa, que implique el reconocimiento de ejemplares presentes en la zona

- Desarrollar habilidades de observación y reconocimiento de ejemplares vegetales que faciliten la enseñanza de la botánica en dicha institución

- Implementar actitudes de respeto, comprensión y conservación por parte de los estudiantes de noveno en la institución educativa a trabajar

\section{METODOLOGÍA}

Como consecuencia a la propuesta que se pretende elaborar, se considera pertinente tener un enfoque cualitativo, dado que lo que se busca indagar es cómo se visualiza la enseñanza de la botánica en la IE, y cualquier producto probablemente será resultado del análisis hacia los estudiantes a partir de ideas previas, identificación del programa académico y posibles estrategias utilizadas por el maestro titular con el que se llevará a cabo la práctica. 
Edición Extra-Ordinaria.

Memorias del 1 Congreso Nacional de Investigación en Enseñanza de la Biología. VI Encuentro Nacional de Investigación en Enseñanza de la Biología y la Educación Ambiental. ISSN 2027 1034. P. p. 495-502.

La INVESTIGACIÓN CUALITATIVA es definida por CERDA (2000) así "las cualitativas hacen parte del grupo de investigaciones "no tradicionales". Aquí la "cualidad" se revela por medio de las propiedades de un objeto o de un fenómeno. La propiedad individualiza al objeto o al fenómeno por medio de una característica que le es exclusiva, mientras que la cualidad expresa un concepto global del objeto $(\ldots)$

Un diseño o investigación de tipo cualitativo, se caracteriza por los siguientes aspectos:

- La interpretación que se le da a las cosas y fenómenos no pueden ser captados o expresados plenamente por la estadística o las matemáticas

- Utiliza preferentemente la inferencia inductiva y el análisis diacrónico en los datos.

- Utiliza los criterios de credibilidad, transferibilidad y confirmabilidad como formas creíbles y confiables de los resultados de un estudio.

- Utiliza múltiples fuentes, métodos e investigadores para estudiar un solo problema o tema, los cuales convergen en torno a un punto central de estudio (principio de triangulación y convergencia)

- Utiliza preferentemente la observación y la entrevista abierta y no estandarizada como técnicas en la recolección de datos

- Centra el análisis en la descripción de los fenómenos y cosas observadas"

Entre las diferentes técnicas que la investigación cualitativa, se resalta la TÉCNICA DESCRIPTIVA en la que CERDA (2000) se presentan las siguientes características.

"- Caracterizar globalmente el objeto de estudio

- Determinar el o los objetos sociales que tienen ciertas características

- Describir el contexto en el cual se presenta cierto fenómeno

- Describir las diferencias que existen (o se dan) entre dos o más subgrupos de una población objeto de estudio 
Bio -grafía Escritos sobre la Biología y su Enseñanza.

Edición Extra-Ordinaria.

Memorias del 1 Congreso Nacional de Investigación en Enseñanza de la Biología.

VI Encuentro Nacional de Investigación en Enseñanza de la Biología y la

Educación Ambiental. ISSN 2027 1034. P. p. 495-502.

- Describir las partes, las categorías o clases que componen el objeto de estudio

- Describir el desarrollo o evolución del objeto de estudio

- Describir las relaciones del objeto de estudio con otros objetos (...)

Una de las funciones principales del método descriptivo es la capacidad para seleccionar las características fundamentales del objeto de estudio y su descripción detallada dentro del marco conceptual de referencia", siendo coherentes con la propuesta trabajada, es este el método de investigación con mayor viabilidad, ya que si se tiene en cuenta que la indagación se realizará a los estudiantes y al profesor titular, la información recolectada puede ser descrita desde diversos aspectos que rodean al individuo.

Dentro de los múltiples estudios que se pueden realizar por medio de este método descriptivo, se rescata el ESTUDIO DE ANÁLISIS DE ACTIVIDADES en el cual, a partir de la revegetalización y posterior conservación de las plantas endémicas se determina que tan posible es esa enseñanza de la botánica a partir del análisis de esta y otras actividades que se plantean a lo largo de la realización de la práctica pedagógica, tales como ideas previas, encuestas, dibujos, relatos, etc.

\section{RESULTADOS Y DISCUSIÓN}

La reformulación del plan de estudios propuestos para el grado Noveno y la actualización docente, a partir de la incorporación de conceptos botánicos son una forma de reconocimiento de aquella contribución que la presente investigación busca en la Institución Educativa. Pues se incorporan ciertos conceptos botánicos al programa propuesto sin dejar de lado los estándares para el área de Ciencias Naturales. Así mismo se evidencia que la profesora titular para el grado Noveno (Clara Possos) incorpora en las clases de ciencias temáticas que son de interés para la población, además de ejemplificando con animales y plantas.

La contextualización de la población escogida, permite indagar sobre posibles problemáticas que facilitan la investigación en el aula, además de ratificar la falencia en el currículo sobre temáticas de tipo botánico. Resultado de dicha investigación se evidencian errores conceptuales que son trabajados en los tópicos del plan de estudios, generando la reorganización del mismo a lo largo del año lectivo, ejemplos de éste es la generalización de la temática de sistema nervioso a trabajarse irritabilidad en el que se incluyen nastias y tropismos en plantas. 
Edición Extra-Ordinaria.

Memorias del I Congreso Nacional de Investigación en Enseñanza de la Biología.

VI Encuentro Nacional de Investigación en Enseñanza de la Biología y la

Educación Ambiental. ISSN 2027 1034. P. p. 495-502.

La incorporación de dichos temas, generan un pequeño cambio en la visión de los estudiantes, pues permite relacionar a las plantas como organismos vivos, ya que se tiene la tendencia a recordar animales con una mayor facilidad. Estas actitudes son observables en tanto la población objeto empieza a reconocer a las plantas como un organismo con un valor intrínseco, además de la importancia de su conservación.

Sin ser el foco de la investigación, se realiza un aporte a otras disciplinas en términos del desarrollo de habilidades y la ubicación espacio-temporal por medio de mapas que los estudiantes realizan para la posterior revegetalización.

Finalmente, se obtiene un recurso educativo (ANEXO 1: Revista Botécnica: Estudios sobre la enseñanza de la botánica en el ITIT), en el que los estudiantes elaboran artículos científicos y otros escritos sobre 5 temáticas botánicas: Histotecnia, morfología, fisiología, restauración ecológica y taxonomía.

\section{CONCLUSIONES}

La enseñanza de la botánica en las instituciones educativas permite que los estudiantes reconozcan a las plantas como organismos vivos, por lo que se le atribuye un valor intrínseco por el simple hecho de existir, se reconoce la importancia de su conservación pues mantienen un equilibrio biológico en cualquier ecosistema del mundo.

La actualización docente en la Institución Educativa asegura que las generaciones que están en formación incorporen los cambios y las novedades en la materia a enseñar, este es el caso de la diferenciación entre planta y vegetal, teniendo en cuenta que la denominación vegetal se le aporta a niveles de organización celular, mientras que la planta se le atribuye a una categoría taxonómica.

La incorporación de conceptos botánicos en el plan de estudios, asegura que día a día se reformule la visión de los estudiantes a estos organismos, por lo que se considera de vital importancia realizar este ejercicio en los diferentes curriculum de las instituciones educativas del país.

Por último, se reconoce que la práctica pedagógica es un espacio que permite una reflexión por parte de los docentes en formación, además de realizar un pequeño aporte a las instituciones de nuestro país.

\section{AGRADECIMIENTOS}

Aunque fueron muchas las personas e instituciones que permitieron realizar esta investigación son de resaltar: 
Edición Extra-Ordinaria.

Memorias del 1 Congreso Nacional de Investigación en Enseñanza de la Biología. VI Encuentro Nacional de Investigación en Enseñanza de la Biología y la Educación Ambiental. ISSN 2027 1034. P. p. 495-502.

La línea de Investigación Enseñanza y Aprendizaje de la botánica que permitió el desarrollo de la investigación y que brindó los espacios para el buen desarrollo del mismo.

A la Profesora Nubia Ladino Ospina que con sus aportes facilitó y orientó la presente investigación

A la Profesora Clara Possos que con su apoyo y colaboración hizo posible este resultado.

A los estudiantes del grado Noveno del ITIT por abrir las puertas de su vida para conocer un poco más el contexto de la población estudiantil.

\section{BIBLIOGRAFÍA}

- ÁLVAREZ.E y ARIAS. H. (1998) "El aprendizaje de algunos conceptos fundamentales en el campo de la botánica. Una experiencia investigación aula”. En: Revista Ciencia y Tecnología. Vol. №14. Bogotá, D.C. Universidad Pedagógica Nacional. Pág. 69 -73.

- ASOCIACIÓN NATURAEDUCA. (S.F.). Recuperado el día 21 de Noviembre de 2010 de http://www.natureduca.com/conserva_conceptos1.php

- CABEZAS. M. Et.al. (2008) "Dosel de tres especies forestales y su relación con la adaptación a suelos degradados por erosión" en: Revista científica U.D.C.A. Vol. № 11. № 2. Bogotá. D.C.

- CERDA. H. (2008) "Los tipos de investigación" en: Los elementos de la investigación. Bogotá. D.C. Editorial Buho. S.A.

- CRUZADA POR LOS BOSQUES Y EL AGUA (S.F). Recuperado el día 21 de Noviembre de 2010 de http://cruzadabosquesagua.semarnat.gob.mx/viii.html

- GARZÓN. Y. y GÓMEZ G. (1997). "Descripción del problema y Conclusiones". En: "Exploración del concepto Bosque Nativo. Como instrumento para fomentar actitudes de conservación en Niños de 12-15 años". Bogotá. D.C-. Trabajo de Grado. Universidad Pedagógica Nacional. Facultad de Ciencia y Tecnología. DBI. 
Edición Extra-Ordinaria.

Memorias del 1 Congreso Nacional de Investigación en Enseñanza de la Biología. VI Encuentro Nacional de Investigación en Enseñanza de la Biología y la Educación Ambiental. ISSN 2027 1034. P. p. 495-502.

- HERSHEY D. (1996) "A historical Perspective on problems in botany teaching". En: "The American biology teacher". Vol 58 NN$^{\circ} 6$. USA.

- JESSUP. M. Et.al. (1999) "El proyecto Curricular de la Licenciatura y la misión". En: Licenciatura en biología. Proyecto curricular. Bogotá. D.C Facultad de Ciencia y tecnología. DBI

- LADINO. N. (2010) Línea de Investigación Enseñanza y aprendizaje de la botánica. Documento de circulación interna. Universidad Pedagógica Nacional.

- LUCIO. R. Enseñanza y didáctica, Educación y Pedagogía, diferencias y relaciones.

- MARTÍNEZ. M. Et.al (1982). Introducción general a la botánica. Universidad del Rosario. Facultad de C. bioquímicas y C. Farmacéuticas.

- SECRETARIA DISTRITAL DE BOGOTÁ (S.F.). Recuperado el día 21 de Noviembre de 2010, de http://webcache.googleusercontent.com/search?q=cache:sXCJNmGyxn0J: www.carlosvicentederoux.org/apc-aa files/f459e34b03aa8797ced4af9d0b6d1b86/Revegetalizaci\%C3\%B3n\%252 0de\%2520los\%2520cerros\%2520con\%2520especies\%2520nativas.doc+R EVEGETALIZACION\&cd=6\&hl=es\&ct=clnk\&gl=co

- UNIVERSIDAD PEDAGÓGICA NACIONAL. (2008) "La Misión". En: Proyecto Político Pedagógico. Bogotá. Cuarta reimpresión.

- VARGAS. G. De la enseñanza a la pedagogía. Revista Colombiana en Educación. Bogotá: Universidad Pedagógica Nacional-CIUP. Págs 61-82 